\title{
Perfil dos idosos usuários de via alternativa de alimentação reinternados em hospital público
}

\author{
Monise Laira do Valle Gonçalves*, Gabriela Aparecida Fabbri Broglio**, \\ Aline Cristina Lozano ${ }^{* * *}$, Neuseli Marino Lamari ${ }^{* * *}$
}

\section{Resumo}

Atualmente, há mudança no perfil epidemiológico e demográfico da população brasileira, com aumento significativo da população idosa. A idade avançada contribui para a suscetibilidade a doenças e maior probabilidade de morte, os idosos compõem o extrato populacional que mais utiliza os serviços de saúde, dos quais se destacam as internações hospitalares caracterizadas por períodos prolongados e maiores índices de readmissão. Com o envelhecimento, muitas alterações fisiológicas vão ocorrendo, inclusive alteração na deglutição, chamada de presbifagia, fator de risco à disfagia. Este estudo objetivou caracterizar o perfil dos idosos reinternados em hospital público usuários de via alternativa de alimentação, por meio de aplicação de questionário desenvolvido por residentes multiprofissionais em Reabilitação Física da Faculdade de Medicina de São José do Rio Preto (Famerp), aplicado a 544 pacientes reinternados nas enfermarias do Hospital de Base de São José do Rio
Preto (SP), no período de junho a outubro de 2014. Dos pacientes que participaram do estudo, foram selecionados $86(15,8 \%)$ que são os idosos usuários de via alternativa de alimentação. Os resultados apontam que as afecções de base mais prevalentes são de causas neurológicas, os principais motivos das reinternações são afecções respiratórias e $80 \%$ contavam com a presença de cuidador. Concluiu-se que uma parcela importante de idosos é usuária de via alternativa de alimentação, predominantemente do sexo masculino, acometidos geralmente pelas afecções de base de causas neurológicas. Sugere-se que sejam realizados novos estudos direcionados a este público, visto que esta população está em crescimento e é responsável por grande parte do uso dos serviços de saúde, necessitando de cuidados especializados.

Palavras-chaves: Envelhecimento. Readmissão do paciente. Transtornos de Deglutição. Fonoaudiologia.

* Fonoaudióloga residente multiprofissional em Reabilitação Física pela Faculdade de Medicina de São José do Rio Preto - Famerp, São José do Rio Preto-SP. E-mail: monise.goncalves@hotmail.com

** Fonoaudióloga residente multiprofissional em Reabilitação Física pela Faculdade de Medicina de São José do Rio Preto - Famerp, São José do Rio Preto-SP. E-mail: gabi_broglio@hotmail.com

**** Fonoaudióloga residente multiprofissional em Reabilitação Física pela Faculdade de Medicina de São José do Rio Preto - Famerp, São José do Rio Preto-SP. E-mail: alinelozano.fono@hotmail.com

***** Fisioterapeuta livre-docente em Ciências da Saúde pela Faculdade de Medicina de São José do Rio Preto Famerp, São José do Rio Preto-SP, professora adjunta III-D da Faculdade de Medicina de São José do Rio Preto - Famerp, São José do Rio Preto-SP, na graduação e pós-graduação lato e stricto sensu. E-mail: neuseli@ famerp.br

Endereço para correspondência: Rua Candido Ferreira de Andrade, 206, IV Centenário, 14960-000, Novo Horizonte, São Paulo, Brasil.

$\rightarrow$ http://dx.doi.org/10.5335/rbceh.v12i1.4548

Recebido em: 18/12/2014. Aceito em: 20/05/2015 


\section{Introdução}

Atualmente, percebe-se a mudança do perfil epidemiológico e demográfico da população brasileira, com o aumento significativo da população idosa, aqueles com sessenta anos ou mais. Em 2010, a população idosa no Brasil configurava um contingente de 23 milhões, correspondente a $11 \%$ da população total, conforme dados do Instituto Brasileiro de Geografia e Estatística (IBGE, 2010).

A idade avançada contribui para a suscetibilidade a doenças e maior probabilidade de morte, assim, o idoso é vulnerável no aspecto biológico (PAZ; SANTOS; EIDT, 2006). O rápido crescimento da população idosa leva a maior demanda dos serviços de saúde, o que requer profissionais preparados para cuidar desse grupo etário (ALMEIDA et al., 2008). Quando existe a necessidade de hospitalização do idoso, espera-se que essa não tenha longa duração, e que ele e sua família recebam orientações para o cuidado domiciliar (MEIRELES et al., 2010).

Dessa forma, idosos têm sido referenciados como o extrato populacional que mais utiliza os serviços de saúde (IBGE, 2010), dos quais destacamos as internações hospitalares caracterizadas por períodos prolongados e com maiores índices de readmissão - uma em cada cinco (HO et al., 2006).

A redução do número de reinternações teria o mérito de não apenas melhorar a capacidade resolutiva do hospital, mas também evitar o sofrimento, os transtornos, a preocupação e os inconvenientes para o paciente e sua família
(DELGALLO; LOPES; MACHARELLI, 2013).

Com o avanço da idade muitas alterações fisiológicas ocorrem, pois o envelhecimento pode ocasionar mudanças na deglutição, chamada de presbifagia, que pode levar à ocorrência de disfagia, estudos sugerem que mais de $20 \%$ dos idosos apresentam esta queixa (FEIJÓ; RIEDER, 2003; RUSSO, 2004).

Disfagia, uma das alterações fonoaudiológicas que mais solicita a presença do fonoaudiólogo à beira do leito, é considerada um déficit de alta morbidade, mortalidade e custos. Está relacionada com consequências graves como a desidratação, a desnutrição e a perda de peso, a aspiração, a pneumonia e a morte (BOURS et al., 2009; CICHERO; HEATON; BASSETT, 2009; SCHINDLER et al., 2008). Por isso, há necessidade de rastrear e identificar a presença de disfagia com adequada avaliação clínica da deglutição, que é realizada de maneira segura, não invasiva, rápida, com baixo custo, reproduzível e com poucos recurSos (ALTMAN; YU; SCHAEFER, 2010; CARNABY-MANN; LENIUS, 2008).

Quanto mais precoce for realizada essa identificação e o início da intervenção fonoaudiológica para reintrodução da alimentação por via oral de maneira segura, maiores serão as chances de melhora do quadro, com consequente diminuição do tempo de internação, de futuras reinternações e dos custos hospitalares (FURKIM; SACCO, 2008).

Assim, define-se como objetivo para este estudo a caracterização do perfil dos idosos reinternados em hospital público usuários de via alternativa de alimen- 
tação, a fim de conhecer essa população mais afundo para trabalhar satisfazendo sua demanda.

\section{Método e casuística}

Trata-se de um estudo quantitativo e descritivo, no qual os dados foram coletados por meio de entrevista semiestruturada, para pacientes ou acompanhantes, em todas as enfermarias, e um questionário que foi aplicado no período de junho a outubro de 2014, em pacientes reinternados em um Hospital-Escola Público de São José do Rio Preto após aprovação pelo Comitê de ética da Faculdade de Medicina de São José do Rio Preto (Famerp) (protocolo 30716814800005415).

Foram considerados como pacientes reinternados aqueles com mais de uma internação decorrente da mesma afecção de base no período de quatro anos. Os participantes da pesquisa foram devidamente esclarecidos sobre os objetivos do estudo e assinaram o termo de consentimento livre e esclarecido.

Participaram do estudo 544 pacientes, de ambos os sexos, adultos ou idosos, com doenças crônicas, reinternados, e que concordaram em participar da pesquisa. Quando o paciente não apresentava condições de responder ao questionário, este foi realizado com o acompanhante, de ambos os sexos, maiores de dezoito anos. Foram excluídos pacientes durante o período de permanência em Unidade de Terapia Intensiva ou que se recusaram a participar da pesquisa.

A coleta de dados foi realizada por meio da aplicação de instrumento ela- borado pelas pesquisadoras na forma de questionário para a caracterização da amostra, contendo dados quanto ao gênero, à idade, ao uso de via alternativa de alimentação, especialidade médica e afeç̧ão de base, motivo da reinternação, presença de cuidador, ventilação mecânica, uso de traqueostomia, uso de espessante alimentar, pneumonia aspirativa, condição motora no leito, perda de peso e alterações emocionais/comportamentais.

Os dados foram tabulados e analisados de forma estatística descritiva simples. Foi utilizado processo de validação por dupla digitação em planilhas do aplicativo Microsoft Excel. Uma vez corrigidos os erros de digitação, os dados foram exportados e analisados no programa Statistical Package for Social Science (SPSS) Software versão 15.0.

\section{Resultados}

O estudo incluiu 544 pacientes, observou-se média de idade de sessenta anos, sendo $314(57,7 \%)$ do sexo masculino. A maioria casada, $270(49,6 \%)$, procedentes da região de São José do Rio Preto, 204 (37,5\%), com escolaridade até a $4^{\mathrm{a}}$ série do ensino fundamental, $235(43,2 \%)$, aposentados, 254 (46,7\%), e moravam com até três pessoas em suas casas, $365(67,1 \%)$. As respostas à entrevista foram informadas pelo próprio paciente, $267(49,1 \%)$, pelos familiares, $256(47,0 \%)$, ou por outras pessoas que estavam acompanhando a internação, $21(3,9 \%)$.

Dos participantes do estudo, 297 $(54,5 \%)$ têm sessenta anos ou mais, e $135(24,8 \%)$ são usuários de via alter- 
nativa de alimentação, porém, para o estudo, foram eleitos os que possuíam as duas características, ou seja, 86 (15,8\%) idosos disfágicos. Observa-se que $63,7 \%$ dos usuários de via alternativa de alimentação são idosos. Foi observado o predomínio do sexo masculino dos pacientes, 51 (61,4\%), com média de idade de 75,04 $\pm 9,39$ anos, a idade mínima foi de sessenta e a máxima de 99 anos.

As afecções de base que mais acometeram esta população foram: Acidente Vascular Encefálico (AVE), em dezenove idosos (22,0\%), Afecções Cardíacas, em treze $(15,1 \%)$, Câncer, onze $(12,7 \%)$, Alzheimer, dez $(11,6 \%)$, outras causas neurológicas, sete $(8,1 \%)$, Cirrose, cinco $(5,8 \%)$, Doença Pulmonar Obstrutiva Crônica (DPOC), quatro $(4,6 \%)$, e fraturas, três $(3,4 \%)$. Os principais motivos pelas reinternações (internação atual) foram afecções respiratórias, $42(48,8 \%)$, afecções urinárias, dezesseis $(18,6 \%)$, e afecções cardíacas, cinco $(5,8 \%)$.

Figura 1 - Exemplificação das afecções de base encontradas na população de idosos deste estudo

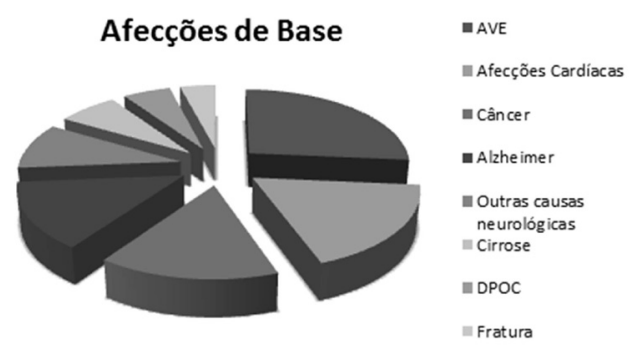

Fonte: elaboração das autoras.

A presença de cuidador foi verificada no caso de 69 idosos $(80,0 \%)$, sendo 57 (66,0\%) filhos, 23 (26,7\%) cônjuges, e um grupo menor $(7,3 \%)$ formado por outros parentes e/ou cuidadores profissionais.

Frente ao quadro clínico, recursos e necessidades destes pacientes, foi verificado que $32(37,2 \%)$ já foram submetidos à ventilação mecânica, e nove $(10,4 \%)$ passaram pelo processo cirúrgico para realização de traqueostomia.

Quanto às dificuldades encontradas durante a alimentação, a maioria dos idosos referiu engasgo, 33 (38,3\%), seguido de tosse, 27 (31,3\%), e um número menor $(30,4 \%)$ se dividiu em odinofagia, escape nasal e sensação de alimento parado. Foi verificado que apenas quatorze $(16,2 \%)$ pacientes fizeram uso de espessante alimentar para adequação da consistência a uma alimentação segura. A perda de peso, durante a internação ou nos últimos três meses, estava presente em $58(67,4 \%)$ pacientes.

Em relação à condição motora, 51 (59,3\%) encontravam-se acamados, 23 $(26,7 \%)$ estavam semidependentes, e doze $(13,9 \%)$ mantinham sua independência. Também foram questionados quanto a alterações emocionais e/ou comportamentais durante a hospitalização, sessenta $(70,0 \%)$ deles referiram alguma alteração.

\section{Discussão}

Segundo dados do Sistema de Informações Hospitalares do Sistema Único de Saúde (SIH-SUS) DE 2001, os idosos representaram $18,3 \%$ das hospitalizações (LOYOLA FILHO et al., 2004 ), porcentagem superior aos $15,8 \%$ encontrados nesta pesquisa. Isso pode ser justificado pelo fato de que o grupo 
estudado restringiu-se apenas aos idosos que faziam uso de via alternativa de alimentação, mostrando-se a grande prevalência desses usuários no contexto hospitalar e enfatizando a importância do tratamento do idoso disfágico.

A reabilitação bem sucedida da disfagia somente será eficaz se o trabalho realizado for interdisciplinar, com atendimentos de fonoaudiólogo, médico, nutricionista, fisioterapeuta, psicólogo, enfermeiro e terapeuta ocupacional. Destaca-se que o principal ganho é para o paciente, pois ele passa a ser visto de forma integral, e as ações realizadas terão por meta facilitar sua inserção na sociedade de modo a melhorar sua qualidade de vida (PEDROSO; SOUSA; SALLES, 2011).

O custo dos serviços de saúde para o idoso é especialmente alto em diversos países do mundo e, no Brasil, não é diferente, isto se dá principalmente por conta da alta taxa de internação hospitalar que ocorre nesta faixa etária (NUNES, 2004). Nesse sentido, a Política Nacional do Idoso (2006) enfatiza que a prática de cuidados às pessoas idosas exige uma abordagem global, interdisciplinar e multidimensional, que considere a interação entre os fatores físicos, psicológicos e sociais que influenciam a saúde dos idosos, e a importância do ambiente no qual eles estão inseridos (BRASIL, 2006).

No presente estudo, a idade média dos pacientes verificada foi de $75,04 \pm 9,39$ anos, a menor idade foi de sessenta, e a maior de 99 anos, resultado próximo ao do estudo de Siqueira et. al. (2004), com um grupo de 94 idosos hospitalizados que apresentaram a média de idade de $78,34 \pm 8,08$ anos, sendo a menor idade 65 , e a maior 99 anos.

Com relação ao gênero, Sales e Santos (2007) registraram estudo no qual o sexo masculino $(72,6 \%)$ superou o feminino $(27,3 \%)$ na representatividade das internações de idosos, assim como no presente trabalho se observou o mesmo predomínio. Papaléo Netto, Carvalho e Sales (2005) destacam que as mulheres têm uma atitude mais cuidadosa em relação à doença, são mais atentas ao aparecimento de sintomas, e utilizam mais os serviços de saúde do que os homens no âmbito de prevenção, nesse sentido há menor representação nas internações hospitalares.

Segundo dados do Sistema de Informações Hospitalares do Sistema Único de Saúde (SIH-SUS) de 2001,, as principais causas de internação entre os idosos foram insuficiência cardíaca e doenças respiratórias (CASTRO; TRAVASSOS; CARVALHO, 2002), achados que corroboram parcialmente os dados desta pesquisa, já que os principais motivos de internações vistos foram as afecções respiratórias $(48,8 \%)$, seguidas das urinárias (18\%), no qual as afecções de base cardíacas aparecem em menor quantidade $(5,8 \%)$.

Conforme dados do Sistema Único de Saúde (SUS), 80\% da população idosa apresenta pelo menos uma doença crônica, e 33,0\% apresenta três ou mais agravos crônico-degenerativos de longa duração, limitações funcionais e necessidade de tratamento pautado na reabilitação. 
Sabe-se que os idosos com maiores taxas de internação hospitalar são aqueles com maior número de doenças crônicas, pior estado funcional, mais restrições das atividades habituais, e que estiveram acamados por motivo de saúde (LOYOLA FILHO et al., 2004), dados que corroboram os dados obtidos neste estudo, 59\% dos idosos eram acamados e $26,7 \%$ semidependentes.

Idosos são vistos socialmente como seres frágeis e incapazes, desconhecedores do que é melhor para si, especialmente em situação de doença e internação, muitas vezes as famílias tomam as decisões por eles, sem ao menos consultá-los, mesmo que tenham condições de decidir (ALMEIDA; AGUIAR, 2011). Ante o exposto, fica claro que o adoecimento e a hospitalização geram grande impacto no aspecto social e psicológico do idoso, o que pode justificar o grande número (70\%) que referiu algum tipo de alteração comportamental e/ou emocional durante este estudo.

Os idosos representavam $11,6 \%$ da população do estado de São Paulo em 2010, mas respondem por $23,8 \%$ das internações do SUS/SP no mesmo ano, e por $31,1 \%$ do valor pago por estas, demonstrando a importância do conhecimento mais pormenorizado da assistência hospitalar a esta faixa etária no SUS (MENDES, 2011).

Este estudo permitiu caracterizar o perfil dos idosos reinternados em um hospital escola público no município de São José do Rio Preto-SP, que fizeram uso de via alternativa de alimentação, objetivando contribuir para a adequação dos serviços prestados para esse grupo populacional e ressaltar a importância da fonoaudiologia inserida neste contexto.

\section{Conclusão}

Observa-se, com base nos dados obtidos no presente estudo, que uma parcela importante de idosos são usuários de via alternativa de alimentação, predominantemente do sexo masculino, acometidos geralmente pelas afecções de base de causas neurológicas. Os principais motivos pelas reinternações hospitalares são afecções respiratórias, a maioria contava com a presença de cuidador e referiu alguma alteração emocional/ comportamental.

Tais achados sugerem a necessidade de investir em melhorias na qualidade do atendimento hospitalar, com maior atuação do fonoaudiólogo na assistência direta ao paciente nos serviços. Sugere-se que sejam realizados novos estudos na área, uma vez que esta população está em constante crescimento e é responsável por grande parte do uso dos serviços de saúde, necessitando de cuidados especializados.

\section{Profile of elderly people who were readmitted public hospital users an alternative route of feeding}

\section{Abstract}

There are currently changing epidemiologic and demographic Brazilian population profiles with significant increase in elderly. The old age contributes to susceptibility to disease and bigger probability of death, the elderly make up the population stra- 
tum highest use of health services, among which are hospital admissions characterized by long days and higher readmission rates. With the aging, many physiological changes have been occurring, including swallowing disorders, presbifagia call and dysphagia risk factors. This study aimed to characterize the profile of readmitted elderly in public hospitals that uses alternative feeding route. Through questionnaire application developed by multidisciplinary Residents in Physical Rehabilitation of Famerp, 544 readmitted patients of Hospital de Base de São José do Rio Preto in the period of June to October 2014. The study included 544 patients. After that, it was selected $86(15.8 \%)$ that are alternative feeding route elderly users, $61 \%$ male, average age of $75.04 \pm 9.39$ years, the most basis conditions of neurological causes, the main reason for readmissions are respiratory conditions, $80 \%$ had the presence of a caregiver and $70 \%$ of them reported any emotional/behavioral change. It was concluded that important portion of seniors are feeding alternative route of users, predominantly male, usually affected by underlying disorders of neurological causes, that suggests new studies because of the population growth and they also are responsible for a great part of health services, requiring skilled care.

Keywords: Human Aging. Patient Readmission. Deglutition Disorders. Speech, Language and Hearing Sciences.

\section{Referências}

ALMEIDA, A. B. A.; AGUIAR, M. G. G. O cuidado do enfermeiro ao idoso hospitalizado: uma abordagem bioética. Revista Bioética, Feira de Santana-BA, v. 19, n. 1, p. 197-217, mar. 2011.

ALMEIDA, M. A. et al. Diagnóstico de enfermagem e intervenções prevalentes no cuidado ao idoso hospitalizado. Revista Latino
Americana de Enfermagem, Porto Alegre, v. 16, n. 4, p. 707-711, jul. 2008.

ALTMAN, K. W.; YU, G. P.; SCHAEFER, S. D. Consequence of Dysphagia in the Hospitalized Patient. Archives of Otolaryngology - Head and Neck Surgery, New York, v. 136, n. 8, p.784-789, Aug. 2010.

BOURS, G. J. J. W. et al. Bedside screening tests VS. Videofluoroscopy or fiberoptic endoscopic evaluation of swallowing to detect dysphagia in patients with neurological disorders: systematic review. Journal of Advanced Nursing, v. 65, n. 3, p.477-493, Oct. 2009.

BRASIL. Ministério da Saúde. Portaria $\mathrm{n}^{\mathrm{o}}$ 2.528, de 19 de outubro de 2006. Aprova a Política Nacional de Saúde da Pessoa Idosa. Diário Oficial da União, Brasília, v. 1, p. 142-5, out. 2006.

CARNABY-MANN, G.; LENIUS, K. The bedside examination in dysphagia. Physical Medicine \& Rehabilitation Clinics of North America, Gainesville, v. 19, p. 747-68, Oct. 2008.

CASTRO, M. S. M.; TRAVASSOS, C.; CARVALHO, M. S. Fatores associados às internações hospitalares no Brasil. Ciência e Saúde Coletiva, Rio de Janeiro, v. 7, n. 4, p. 795-811, jun. 2002.

CICHERO, J. A. Y.; HEATON, S.; BASSETT, L. Triaging dysphagia: nurse screening for dysphagia in an acute hospital. Journal of Clinical Nursing, Brisbane, v. 18, p. 16491659, jun. 2009.

DELGALLO, A. P. M.; LOPES, E. S.; MACHARELLI, C. A. Reinternações no Hospital Estadual Bauru: ocorrência e possíveis causas. Saúde Coletiva, Bauru-SP, v. 59, n. 10, p. 26-30, 2013.

FEIJÓ, A. V.; RIEDER, C. R. M. Distúrbios da deglutição em idosos. In: JACOB, J. S.; LEVY, D. S.; SILVA, L. M. C. (Org.). Disfagia: avaliação e tratamento. São Paulo, 2003. p. 225-232. 
FURKIM, A. M.; SACCO, A. B. F. Eficácia da fonoterapia em disfagia neurogênica usando a escala funcional de ingestão por via oral (fois) como marcador. Revista CEFAC, São Paulo, v. 10, n. 4, p. 503-12, out. 2008.

$\mathrm{HO}, \mathrm{K}$. M. et al. C-reactive protein concentration as a predictor of intensive care unit readmission: a nested case-control study. Journal of Critical Care, Perth, v. 21, n. 3, p. 259-265, Sept. 2006.

IBGE - INSTITUTO BRASILEIRO DE GEOGRAFIA E ESTATÍSTICA. Síntese de indicadores sociais Uma análise das condições de vida da população brasileira 2010. Rio de Janeiro: IBGE, 2010. Disponível em: <http://www.ibge.gov.br/home/estatistica/ populacao/condicaodevida/indicadoresminimos/sinteseindicsociais2010/SIS_2010.pdf>. Acesso em: 10 nov. 2014.

LOYOLA FILHO, A. I. et al. Causas de internações hospitalares entre idosos brasileiros no âmbito do Sistema Único de Saúde. Epidemiologia e Serviços de Saúde, Belo Horizonte, v. 13, n. 4 , p. 229-238, out. 2004.

MEIRELES, V. C. et al. Autonomia e direito à informação: contribuições para a gestão do cuidado de idosos hospitalizados. Ciencia y Enfermeria XVI, Maringá, v. 16, n. 2, p. 5968, jun. 2010.

MENDES, J. D. V. Internações de idosos no SUS/SP em 2010. Boletim Eletrônico GAIS, São Paulo, a. 3, n. 8, set. 2011. (Publicação do Grupo Técnico de Avaliação e Informações de Saúde; Coordenadoria de Planejamento de Saúde - CPS/SES/SP).

NUNES, A. O envelhecimento populacional e as despesas do Sistema Único de Saúde. In: CAMARANO, Ana Amélia (Org.). Os novos idosos brasileiros: muito além dos 60? Rio de Janeiro: IPEA, 2004. p. 427-450.

PAPALÉO NETTO, M.; CARVALHO, E. T. F.; SALLES, R. F. N. Fisiologia do envelhecimento. In: CARVALHO FILHO, E. T.; PAPALÉO NETTO, M. (Org.). Geriatria: fundamentos, clínica e terapêutica. 2. ed. São Paulo: Atheneu, 2005. p. 43-62.

PAZ, A. A.; SANTOS, B. R. L. D.; EIDT, O. R. Vulnerabilidade e envelhecimento no contexto da saúde. Acta Paulista de Enfermagem, São Paulo, v. 19, n. 3, p. 338-342, set. 2006.

PEDROSO, C. G. T.; SOUSA, A. A.; SALLES, R. K. Cuidado nutricional hospitalar: percepção de nutricionistas para atendimento humanizado. Ciência \& Saúde Coletiva, Florianópolis, v. 16, n. 1, p. 1155-1162, out. 2011.

RUSSO, I. P. Intervenção fonoaudiológica na terceira idade. Rio de Janeiro: Revinter, 2004.

SALES, F. M.; SANTOS, I. Perfil de idosos hospitalizados e nível de dependência de cuidados de enfermagem: identificação de necessidades. Texto Contexto - Enfermagem, Florianópolis, v. 16, n. 3, p. 495-502, jul. 2007.

SCHINDLER, A. et al. Rehabilitative management of Oropharyngeal Dysphagia in Acute Care Settings: data from a large italian teaching hospital. Dysphagia, Milan, v. 23, p. 230-236, Oct. 2008.

SIQUEIRA, A. B. et al. Impacto funcional da internação hospitalar de pacientes idosos. Revista Saúde Pública, São Paulo, v. 38, n. 5, p. 687-694, maio 2004. 